\title{
SOX2 Mediates Carbon Nanotube-Induced Fibrogenesis and Fibroblast Stem Cell Acquisition
}

\author{
Chayanin Kiratipaiboon, ${ }^{\dagger}$ Maria Voronkova,${ }^{\ddagger}$ Rajib Ghosh,${ }^{\dagger}$ Liying W. Rojanasakul, ${ }^{\perp}$ \\ Cerasela Zoica Dinu, ${ }^{\S}$ Yi Charlie Chen, ${ }^{\Uparrow}$ and Yon Rojanasakul ${ }^{*},+\neq$
}

tDepartment of Pharmaceutical Sciences, West Virginia University, Morgantown, West Virginia 26506, United States

fWVU Cancer Institute, West Virginia University, Morgantown, West Virginia 26506, United

States

${ }^{\perp}$ Health Effects Laboratory Division, National Institute for Occupational Safety and Health, Morgantown, West Virginia 26505, United States

\$Department of Chemical and Biomedical Engineering, West Virginia University, Benjamin M.

Statler College of Engineering and Mineral Resources, Morgantown, West Virginia 26506, United States

"College of Science, Technology and Mathematics, Alderson Broaddus University, Philippi, West Virginia 26416, United States

4 pages, 2 Tables, 3 Figures

${ }^{*}$ Corresponding Author

Address correspondence to: Yon Rojanasakul, West Virginia University, Morgantown, WV, 26506, USA. Phone: 304-293-1476. Fax: 304-293 2576, E-mail: yrojan@hsc.wvu.edu

Table S1. Hydrodynamic diameter and Zeta potential of SWCNT $0.1 \mathrm{mg} \mathrm{mL}^{-1}$ in sterile water and $0.5 \%$ BSA in dPBS 


\begin{tabular}{|l|c|c|}
\hline \multicolumn{1}{|c|}{ Properties } & Sterile water & $\mathbf{0 . 5 \%}$ BSA in dPBS \\
\hline Hydrodynamic diameter $(\mathrm{nm})$ & $207.5 \pm 82$ & $126.1 \pm 69.1$ \\
\hline Zeta potential $(\mathrm{mV})$ & $-12.83 \pm 1.58$ & $-9.88 \pm 1.09$ \\
\hline
\end{tabular}

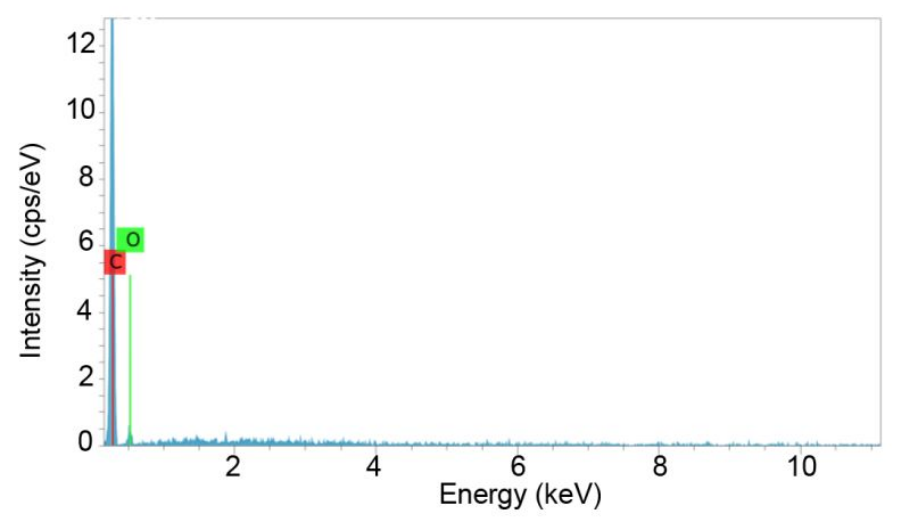

Figure S1. EDS spectra of pristine SWCNTs.

Table S2. Carbon nanotube (CNT) doses for in vitro studies

1 indicates plate format for immunoblotting, anoikis and double immunofluorescence.

2 indicates plate format for sphere formation assay, immunofluorescence and collagen staining

3 indicates plate format for cell proliferation.

\begin{tabular}{|c|c|c|c|c|c|}
\hline Size of culture & $\begin{array}{c}\text { Growth area } \\
\left(\mathrm{cm}^{2}\right)\end{array}$ & $\begin{array}{l}\text { Medium volume } \\
\qquad(\mathrm{mL})\end{array}$ & $\begin{array}{c}\text { CNT surface } \\
\text { dose }\left(\mu \mathrm{g} \mathrm{cm}^{-2}\right)\end{array}$ & CNT mass $(\mu \mathrm{g})$ & $\begin{array}{c}\text { CNT } \\
\text { concentration } \\
\left(\mu \mathrm{g} \mathrm{mL}^{-1}\right)\end{array}$ \\
\hline 6 -well plate ${ }^{1}$ & 9.50 & 2.000 & $0.050-0.2$ & $0.475-1.900$ & $0.237-0.950$ \\
\hline 24-well plate 2 & 1.90 & 0.400 & $0.050-0.2$ & $0.095-0.380$ & $0.237-0.950$ \\
\hline 96-well plate 3 & 0.32 & 0.067 & $0.025-1.6$ & $0.008-0.512$ & $0.119-7.596$ \\
\hline 100 mm plate 4 & 55.00 & 11.600 & $0.050-0.2$ & $2.750-11.000$ & $0.237-0.950$ \\
\hline
\end{tabular}

4 indicates plate format for ALDH activity and chromatin immunoprecipitation. 
(a)

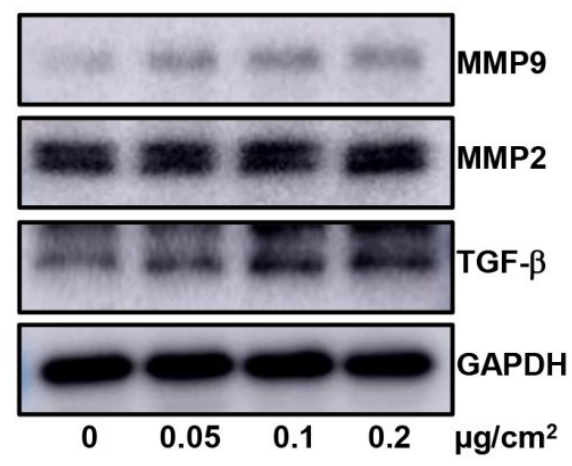

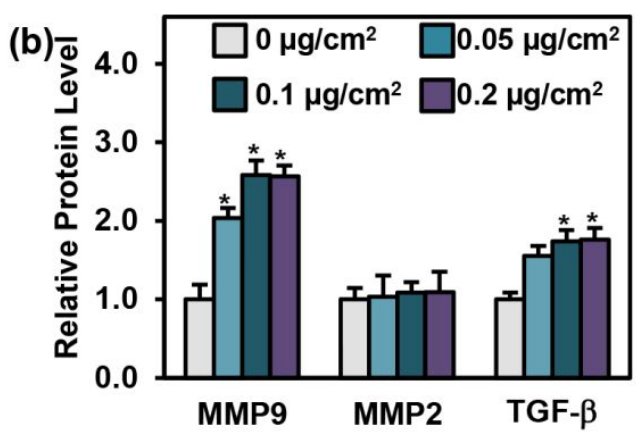

Figure S2. Effects of SWCNT on TGF- $\beta$ and matrix metalloproteinases (MMPs) expression. (a) Western blot analysis of TGF- $\beta$, MMP9 and MMP2. GAPDH was used to confirm equal loading of samples. (b) Quantitative analysis of TGF $-\beta$, MMP9 and MMP2. Data are mean \pm SD $(n=3)$, ${ }^{*} p<0.05$ versus control cells.

(a)
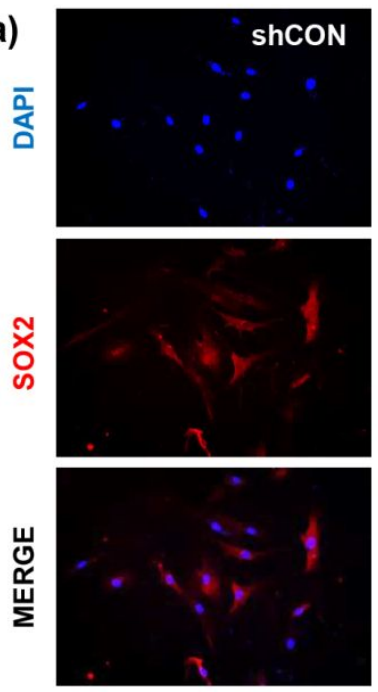
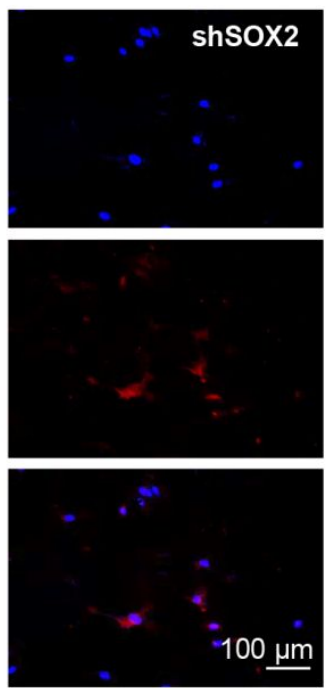

(b)

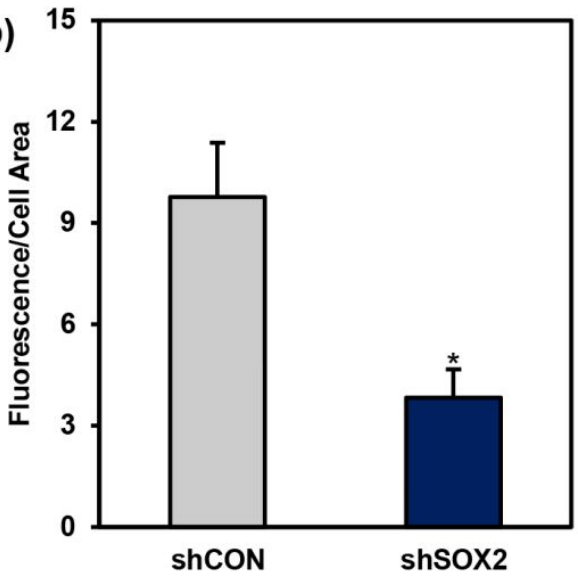

Figure S3. Expression of SOX2 in shCON and shSOX2 cells. (a) Representative immunofluorescence images and (b) quantification of SOX2 (red) in shRNA control (shCON) and SOX2 knockdown (shSOX2) cells. Scale bar is $100 \mu \mathrm{m}$. 100X magnification. Data are mean $\pm \mathrm{SD}(\mathrm{n}=3),{ }^{*} p<0.05$ versus shCON cells. 
(a)

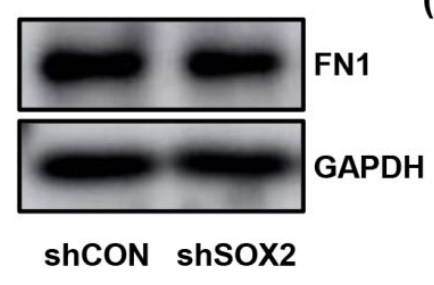

(b)

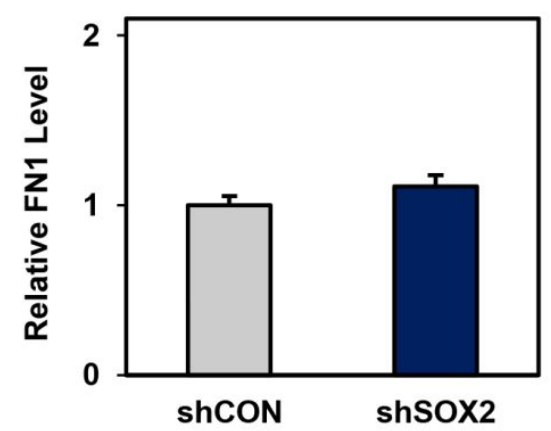

Figure S4. Effect of SOX2 knockdown on fibronectin 1 (FN1) expression in shCON and shSOX2 cells. (a) Western blot images and (b) quantification of FN1 in shCON and shSOX2 cells. Data are mean $\pm S D(n=3)$.

Table S3. Primer sequences used for RT-qPCR

\begin{tabular}{|l|l|l|}
\hline \multicolumn{1}{|c|}{ Gene } & \multicolumn{2}{|c|}{ Sequence (5'-3') } \\
\hline COL1A1 (a) & F: AGTGCAACGCTTACCCTTG & R: CTTCTCTAACCTGGCCTCTTG \\
\hline COL1A1 (b) & F: ACCTAGACATCTTAAAAGCCCG & R: TTGGCTAAAAGTGACCTGGAG \\
\hline COL1A2 (a) & F: CGCCTCCCCAGTTTAAGTAAT & R: GAGACCAGCCTGACCAATATG \\
\hline COL1A2 (b) & F: CCTGGTCCTCTTGGCATTG & R: TGTTGGACTCACATCACGAC \\
\hline
\end{tabular}

